\title{
REDUCIBILITY OF POSITIVE TYPE POLYNOMIALS
}

\section{HENRY TEICHER}

1. Introduction. Let $T_{+}$denote the set of all positive type polynomials, i.e., $T_{+}=\left\{\sum_{j=0}^{m} a_{j} x^{j}\right\}=\left\{P_{m}(x)\right\}$ where $a_{j} \geqq 0,0 \leqq j \leqq m \geqq 1$, $a_{m} \neq 0$. Clearly, $T_{+}$is closed under multiplication but not factorization. An analogous but wider class of functions has been considered by Rosenbloom [4].

Let $T_{i}$ be that subset of $T_{+}$which is irreducible over $T_{+}$(i.e., $P_{m}(x) \in T_{i}, \quad P_{m}(x)=P_{k}(x) \cdot P_{m-k}(x), \quad 0<k<m$, implies $P_{k} \notin T_{+}$or $\left.P_{m-k}(x) \notin T_{+}\right)$. It would be of interest to have a criterion for deciding when an arbitrary member of $T_{+}$lies in $T_{i}$ or $T_{r}=T_{+}-T_{i}$.

For $m=2$, the sign of the discriminant of the quadratic provides the answer while for $m=3$ a NSC that $P_{m}(x) \in T_{r}$ is $a_{1} a_{2} \geqq a_{0} a_{3}$. For $m=4$ a NSC can be given ( $\$ 4)$ in terms of the location of a root of $P_{4}(-x)$ (which is not very satisfactory) from which simpler conditions can be derived in certain cases. Some partial results are given for general $m$.

The preceding is manifestly applicable to the so-called "arithmetic of probability distributions" (initiated by Paul Lévy, see e.g. [2]) provided the random variables take on only a finite number of rational values (or values of the form $a+k b, k$ rational, $a, b$ real). The proof of Theorem 1 contains a method of constructing an indecomposable (prime) distribution for any positive integer $m \geqq 2$.

2. Preliminary considerations. Unless the contrary is stated it will be understood that $P_{m}(x) \in T_{+}$. In the treatment of this polynomial there is no loss of generality in supposing $a_{m}=1, a_{0}>0$. Denoting by $A_{i}, A_{i}^{\prime}, B_{i}, C_{i}$ positive real numbers, the canonical decomposition of $P_{m}(x) \in T_{+}$into quadratic and linear factors can only be of the form

$$
\begin{aligned}
\prod_{j}\left(x^{2}-A_{j} x+B_{j}\right) \prod_{k}\left(x^{2}+A_{k}^{\prime} x+B_{k}\right) \prod_{i}\left(x^{2}+B_{i}\right) \prod_{n}\left(x+C_{n}\right) \\
=\left(\prod_{j} Q_{j}^{-}\right)\left(\prod_{k} Q_{k}^{+}\right)\left(\prod_{i} Q_{i}^{0}\right)\left(\prod_{n} L_{n}^{+}\right) .
\end{aligned}
$$

The number of linear factors $L_{n}^{+}$may be supposed to be at most one.

Let $T_{q} \subset T_{+}$denote the set of positively quadral polynomials (see [3]), i.e., all quadratic factors are of the $Q^{+}$or $Q^{0}$ variety and any linear factors are of the form $L^{+}$. Hurwitz [1] has given a NSC that all roots of $P_{m}(x)$ have negative real part. By inserting a few

Received by the editors November 4, 1953 and, in revised form, July 8, 1954. 
equality signs here and there in I. Schur's proof of Hurwitz's Theorem (see [5, appendix]) one obtains the following elementary extension:

Extended Hurwitz Theorem. A necessary and (if $a_{1} \neq 0$ ) suffcient ${ }^{1}$ condition that $P_{m}(x) \in T_{q}$ is that the determinants

$$
\begin{aligned}
& D_{1}=a_{1}, \quad D_{2}=\left|\begin{array}{ll}
a_{1} & a_{0} \\
a_{3} & a_{2}
\end{array}\right|,
\end{aligned}
$$

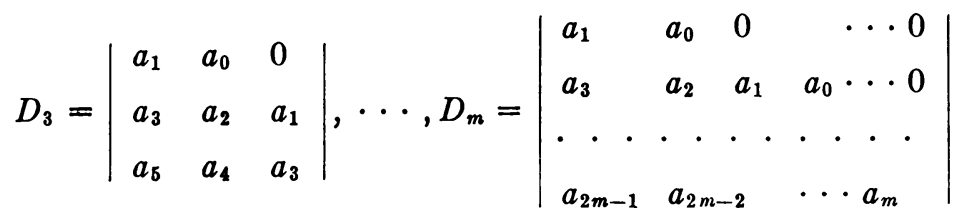

be non-negative. Here $a_{j}=0$ for $j>m$.

Obviously, $T_{q}$ is closed under factorization and $T_{q} \subset T_{r}$. Thus, the problem originally stated retains interest only if the extended Hurwitz criterion is violated, i.e., $P_{m}(x) \in T^{*}=T_{+}-T_{q}$. In such a case there is at least one factor of the $Q^{-}$type and this may be represented by $Q^{-}(x, \epsilon)=x^{2}-A x+\epsilon A^{2}$ where $\epsilon, A>0$. Actually $\epsilon>1 / 4$ if $Q^{-}(x, \epsilon)$ is to have no positive root (be a factor of $P_{m}(x)$ ).

\section{Minimal degree polynomials.}

Theorem 1. A NSC that there exist $P_{m}(x) \in T_{+}$containing $Q^{-}(x, \epsilon)$ $=x^{2}-A x+\epsilon A^{2}$ as a factor is that $m \geqq$ smallest integer $j$ such that $j \operatorname{arc} \cos \left(1 / 2 \epsilon^{1 / 2}\right) \geqq \pi .^{2}$

Necessity. By the transformation $x=A y$, we may suppose $A=1$. Let $\delta=4 \epsilon-1>0$ and $b_{j}=2^{-j} a_{j} \geqq 0$. As the roots of $Q^{-}(x, \epsilon)=0$ are also roots of $P_{m}(x)=0$,

$$
\begin{gathered}
\sum_{j=0}^{m} b_{j}\left[\sum_{k=0}^{[j / 2]}\left(\begin{array}{c}
j \\
2 k
\end{array}\right)(-1)^{k} \delta^{k}\right]=0, \\
\delta^{1 / 2} \sum_{j=1}^{m} b_{j}\left[\sum_{k=0}^{[(j-1) / 2]}\left(\begin{array}{c}
j \\
2 k+1
\end{array}\right)(-1)^{k} \delta^{k}\right]=0 .
\end{gathered}
$$

Denote the coefficients of $b_{j}$ in $(1.1)$ by $f_{j}=f_{j}(\delta)=2^{j-1} \bar{f}_{j}(\epsilon)$ and those in (1.2) by $h_{j}=\delta^{1 / 2} g_{j}=\delta^{1 / 2} g_{j}(\delta)=\delta^{1 / 2} 2^{j-1} \bar{g}_{j}(\epsilon)$. These quantities are virtually the Tchebycheff polynomials of the 1 st and 2 nd kind,

${ }^{1}$ If $a_{1}=0$ and $a_{2 j+1}>0$ for some $j \geqq 1, P_{m} \in T_{q}$; if $a_{2 j-1}=0$ for all $j \geqq 1, P(x) \in T_{q}$ is of even degree and $P_{2 m}(x)=R_{m}\left(x^{2}\right)=R_{m}(y)$ whence if $R_{m}(y) \notin T_{q}, P_{2 m}(x) \notin T_{q}$.

2 The principal value of the arc cosine is to be taken. 
say $T_{j}^{(1)}(x)$ and $T_{j}^{(2)}(x)$. In fact $T_{j}^{(1)}(x)=x^{i} f_{j}\left(\left(1-x^{2}\right) / x^{2}\right)$ and $T_{j}^{(2)}(x)$ $=x^{i} g_{j+1}\left(\left(1-x^{2}\right) / x^{2}\right)$. Letting $1+i \delta^{1 / 2}=r(\cos \theta+i \sin \theta)$ with $r=(1+\delta)^{1 / 2}, 0<\theta<\pi / 2$ we have from DeMoivre's theorem

$$
\begin{aligned}
& f_{j}=f_{j}(\delta)=(1+\delta)^{j / 2} \cos j \theta, \\
& h_{j}=h_{j}(\delta)=(1+\delta)^{j / 2} \sin j \theta=\delta^{1 / 2} g_{j}(\delta) .
\end{aligned}
$$

Rewrite (1.1) and (1.2) as

$$
\sum_{j=0}^{m} b_{j} f_{j}=0=\sum_{j=1}^{m} b_{j} h_{j} .
$$

Since $b_{j} \geqq 0$ (actually $b_{0}>0$ ), from (1.4) and (1.5) there must be at least one non-negative $f_{i}$ and non-positive $h_{i}$ for $i \leqq m$. By (1.3) this requires $j \theta \geqq \pi$, concluding necessity.

For any given $\epsilon(>1 / 4)$, denote by $M=M(\epsilon) \geqq 3$ the minimal (integral) value of $\bar{m}$ for which $\bar{m}$ arc $\cos \left(1 / 2 \epsilon^{1 / 2}\right) \geqq \pi$. Then $P_{M}(x)$ exists and will be called a minimal polynomial containing $Q^{-}$or minimal for $Q^{-}$or simply "minimal for $\epsilon$." As $\epsilon \rightarrow 1 / 4$, the roots of $Q^{-}(x, \epsilon)$ approach the positive real axis and $M(\epsilon) \rightarrow \infty . M(\epsilon)$ is a step function continuous on the right and may be computed from the relation $\epsilon \geqq(1 / 4) \sec ^{2}(\pi / M)$.

Sufficiency. It suffices to construct a $P_{M}(x)$. From (1.1), $f_{0}=1$. Choose $b_{M}=2^{-M}, b_{M-1}=2^{-M}\left|h_{M}\right|\left(h_{M-1}\right)^{-1}$, and $b_{1}=b_{2}=\cdots=b_{M-2}$ $=0$ thus satisfying (1.5). To fulfill (1.4) take $b_{0}=2^{-M}\left[\left|f_{M}\right|-f_{M-1}\right.$ $\left.\left|h_{M}\right|\left(h_{M-1}\right)^{-1}\right]$. From (1.3), $h_{M-1}>0, f_{M-1}<0$ whence $b_{0}>0$. If $A \neq 1, a_{j}=2^{i} A^{-i} b_{j} \geqq 0$. Q.E.D.

Clearly, the minimal degree $M$ although not $P_{M}(x)$ is unique. ${ }^{3} \mathrm{~A}$ less accurate but simpler bound is given by

CoROllary 1.1. For $1 / 4<\epsilon \leqq 1 / 2$, a necessary condition that $Q^{-}(x, \epsilon)$ divides $P_{m}(x)$ is $m \geqq M \geqq$ smallest integer $j$ such that $j \geqq \pi \delta^{-1 / 2}$.

Proof. This follows from arc $\tan \delta^{1 / 2}<\delta^{1 / 2}$ for $0<\delta<1$.

Using the trigonometric identities for $\sin (\theta+\phi)$ and $\cos (\theta+\phi)$ and (1.1), (1.2) we have $f_{j}(\delta)=f_{j-1}(\delta)-\delta g_{j-1}(\delta)$ and $g_{j}(\delta)=g_{j-1}(\delta)$ $+f_{j-1}(\delta), j \geqq 2$, from which it is readily deduced that both $g_{j}(\delta)$ and $f_{j}(\delta)$ satisfy the equation $g_{j}(\delta)=2 g_{j-1}(\delta)-(1+\delta) g_{j-2}(\delta), j \geqq 2$. A more usable form of this equation is (see just below (1.2))

$$
\bar{g}_{j}(\epsilon)=\bar{g}_{j-1}(\epsilon)-\epsilon \bar{g}_{j-2}(\epsilon),
$$$$
j \geqq 2 \text {. }
$$

3 In the special cases $\epsilon=\epsilon_{M}^{*}=4^{-1} \sec ^{2}(\pi / M), M=3,4, \cdots$, the minimal polynomial is unique and of the form $x^{M}+a_{0}$. For from (1.5), $h_{M}=0$ whence also $b_{j}$ $=0,1 \leqq j \leqq M-1$. 
Define now a "residual polynomial"

$$
P_{m-2}(x)=\sum_{j=0}^{m-2} c_{j} x^{j} \text { by } P_{m}(x)=\sum_{j=0}^{m} a_{j} x^{j}=\left(x^{2}-A x+\epsilon A^{2}\right) P_{m-2}(x) .
$$

We have then

Theorem 2. If $Q^{-}(x, \epsilon)$ divides the minimal polynomial $P_{M}(x) \in T_{+}$, the corresponding residual polynomial $P_{M-2}(x) \in T_{+}$and (excluding $\left.c_{M-2}=a_{M}=1\right)$ its coefficients form an increasing sequence.

Proof. Consider at first any $P_{m}(x) \in T_{+}$and as before let $A=1$ $=a_{m}=c_{m-2}$. If we define $c_{-2}=c_{-1}=c_{m-1}=c_{m}=0$, then for any residual polynomial (regardless of minimality)

$$
\epsilon c_{k}-c_{k-1}+c_{k-2}=a_{k}, \quad k=0,1, \cdots, m .
$$

By induction using (1.6) it follows that

$$
\epsilon^{j+1} c_{j}=\sum_{i=0}^{j} \epsilon^{j-i} a_{j-i} \bar{g}_{i+1}(\epsilon), j=0,1, \cdots, m-2 .
$$

If now $m=M(\epsilon)$, then $c_{j} \geqq 0, j=1,2, \cdots, M-2$, since $\bar{g}_{k}>0$ for $k<M$ in view of (1.3). Next from (2.2) and subsequently (1.6),

$$
\begin{aligned}
c_{j+1}-c_{j} & =\epsilon^{-1} a_{j+1}+\sum_{i=0}^{j} \epsilon^{-(i+2)}\left[\bar{g}_{i+2}-\epsilon \bar{g}_{i+1}\right] a_{j-i} \\
& =\epsilon^{-1} a_{j+1}+\sum_{i=0}^{j} \epsilon^{-(i+2)} a_{j-i} \bar{g}_{i+3} \geqq \epsilon^{-(j+2)} a_{0} \bar{g}_{j+3} \geqq 0
\end{aligned}
$$

so long as $j<M(\epsilon)-3$.

Corollary 2.1. If $Q^{-}(x, \epsilon)$ divides $P_{m}(x) \in T_{+}$and $m \leqq 2 M-1$, the residual polynomial $P_{m-2}(x) \in T_{+}$and its coefficients from $c_{0}$ to $c_{M-3}$ form an increasing sequence.

Proof. Suppose $P_{n+r}(x)=\left(x^{2}-x+\epsilon\right) \sum_{j=0}^{n+r-2} \quad c_{j} x^{j}$. As before $c_{0}, c_{1}, \cdots, c_{M-2}$ are non-negative. By a backwards induction using (2.1) and (1.6), $c_{n+j-1}=\sum_{i=j+1}^{r} \bar{g}_{i-j}(\epsilon) a_{n+i}$ for $j=0,1, \cdots, r-1$. The largest subscript of $\bar{g}$ occurs for $i=r, j=0$ giving $\bar{g}_{r}(\epsilon)$. Hence if $n=M$ and $r \leqq M-1$ the coefficients $c_{M-1}, c_{M}, \cdots, c_{M+r-2}$ are nonnegative. Thus $P_{m-2}(x) \in T_{+}$for $m \leqq 2 M-1$. Q.E.D.

If $m \geqq 2 M, P_{m-2}(x)$ need not $\in T_{+}$; e.g., if $M(\epsilon)=M(2)=3$

$$
\left(x^{6}+5 x^{3}+8\right)=\left(x^{2}-x+2\right)\left(x^{4}+x^{3}-x^{2}+2 x+4\right) \text {. }
$$

Denote by $M_{i}=M_{i}\left(\epsilon_{i}\right)$ the degree of a minimal polynomial contain- 
ing $Q_{i}^{-}\left(x, \epsilon_{i}\right)$ as a factor. We consider next some effects of having two $Q^{-}$factors.

Theorem 3. Let $P_{m}(x) \in T_{+}$. If $P_{m}(x)=\left(x^{2}-A_{1} x+\epsilon_{1} A_{1}^{2}\right) P_{m-2}(x)$ with $m=M_{1}+j$ where $j=0$ or 1 and $P_{m-2}(x)=\left(x^{2}-A_{2} x+\epsilon_{2} A_{2}^{2}\right) P_{m-4}(x)$ where necessarily $\epsilon_{1} \leqq \epsilon_{2}$, then $M_{1}>2 M_{2}-1-j$ and $P_{m-2}(x)$ is not minimal for $\boldsymbol{\epsilon}_{2}$.

Proof. Let $P_{m}(x)=\left(x^{2}-A_{2} x+\epsilon_{2} A_{2}^{2}\right) R_{m-2}(x)$. As $m \leqq M_{1}+1$, $R_{m-2}(x) \notin T_{+}$. By Corollary 2.1, $M_{1}+j>2 M_{2}-1$. Furthermore $P_{m-2}(x)$ cannot be minimal for $\epsilon_{2}$ since $M_{1}+j-2=M_{2}$ would imply $3>M_{2}$. Q.E.D.

Theorem 4. If $P_{6}(x)=\sum_{j=0}^{6} a_{j} x^{j}\left(\in T_{+}\right)$is minimal for $Q^{-}\left(x, \epsilon_{1}\right)$, then the residual polynomial $P_{4}(x) \in T_{q}$.

Proof. For suppose $P_{6}(x)=\left(x^{2}-C x+\epsilon_{1} C^{2}\right) P_{4}(x), P_{4}(x)=\left(x^{2}-A x\right.$ $\left.+\epsilon A^{2}\right)\left(x^{2}+B x+D\right)$ with positive $C, A, D, \epsilon$. As $a_{5} \geqq 0$ implies $B>0$, let $D=\mu B^{2}, \mu>0$. As before, take $A=1$. By Theorem 2

$(4.1,4.2,4.3) \quad B-1 \geqq \epsilon+\mu B^{2}-B \geqq B(\epsilon-\mu B) \geqq \epsilon \mu B^{2}$.

By Theorem 3, $P_{4}$ is not minimal for $\epsilon$; hence $M(\epsilon)=3, \epsilon \geqq 1$. Now (4.1) implies $\mu^{-1}-\epsilon-1 \geqq \mu\left(B-\mu^{-1}\right)^{2} \geqq 0$, i.e., $\mu^{-1}-1 \geqq \epsilon \geqq 1$ and in particular $\mu \leqq 1 / 2$. From (4.2), $g(B)=2 \mu B^{2}-(1+\epsilon) B+\epsilon \geqq 0$. In view of $\mu \leqq 1 / 2, g(B)=0$ has two real roots $B_{1} \leqq B_{2}$. Again $\mu \leqq 1 / 2$ insures $B_{1}<1$. As $P_{6}(x) \in T_{+}$implies $B \geqq C+1 \geqq 1$, we must have $B \geqq B_{2}$.

On the other hand from (4.3), $B \leqq \epsilon \mu^{-1}(1+\epsilon)^{-1}$. This contradicts $B \geqq B_{2}$ in view of $(1+\epsilon)(4 \mu)^{-1} \geqq \epsilon \mu^{-1}(1+\epsilon)^{-1}$. Q.E.D. This theorem is not true for general $m$. For example, let $\operatorname{arc} \cos \left(2 \epsilon^{1 / 2}\right)^{-1}=18^{\circ}$, $M(\epsilon)=10$. Then

$$
\begin{aligned}
\left(x^{10}+1\right)= & \left(x^{2}+1\right)\left(x^{2}-2 x \cos 18^{\circ}+1\right)\left(x^{2}-2 x \cos 54^{\circ}+1\right) \\
& \cdot\left(x^{2}+2 x \sin 36^{\circ}+1\right)\left(x^{2}+2 x \sin 72^{\circ}+1\right) .
\end{aligned}
$$

The preceding suggests

Criterion 1. Given $P_{m}(x) \in T^{*}=T_{+}-T_{q}$ (verifiable by the extended Hurwitz theorem), find its canonical decomposition and the $\epsilon_{k}$ corresponding to all quadratic factors of the type $Q_{k}^{-}\left(x, \epsilon_{k}\right)=x^{2}$ $-A_{k} x+\epsilon_{k} A_{k}^{2}$ where $A_{k}>0$. Let $\epsilon=\min _{k} \epsilon_{k}$. If $M(\epsilon)=m, P_{m}(x) \in T_{i}$. Also if $P_{m}(x)$ has no real roots and $M(\epsilon)>m-2, P_{m}(x) \in T_{i .}{ }^{4}$

4. Special cases. For $m=3$, the extended Hurwitz criterion yields $a_{1} a_{2}<a_{0} a_{3}$ as a NSC for $P_{3}(x) \in T_{i}$.

- An obvious sufficient condition that $P_{m}(x) \in T_{i}$ is that all differences of pairs of exponents with nonzero coefficients $a_{j}$ be distinct [2]. 
Consider next the case $m=4, a_{m}=a_{4}=1$. Then if $a_{1} \neq 0$, a NSC that $P_{4}(x) \in T^{*}$ is $a_{3}\left[a_{1} \cdot a_{2}-a_{0} \cdot a_{3}\right]<a_{1}^{2}$. Should $a_{1}=0$, then $a_{3}>0$ or $a_{3}=0$, $a_{2}^{2}<4 a_{0}$ is NS that $P_{n}(x) \in T_{i} \subset T^{*}$.

Define $Q_{2}(x)=x^{2}-a_{3} \cdot x+a_{2}$ with $r_{1} \leqq r_{2}$ the roots of $Q_{2}(x)=0$. Then if $T_{r}^{*}=T_{r}-T_{q}$, we have

Theorem 5. Let $P_{4}(x) \in T^{*}$. If $a_{3}^{2} \geqq 4 a_{2},(<), a N S C$ for $P_{4}(x) \in T_{r}^{*}$ is that $P_{4}(-x)$ have a root in $\left(0, r_{1}\right]$ or $\left[r_{2}, a_{3}\right]$, (in $\left.\left(0, a_{3}\right]\right)$.

Proof. The only possible factorization is $P_{4}(x)=(x+z)\left[x^{3}+\left(a_{3}\right.\right.$ $\left.-z) x^{2}+Q_{2}(z) x+Q_{3}(z)\right]$ where $Q_{3}(z)$ is a cubic polynomial and $P_{4}(-z)=0, \quad 0<z \leqq a_{3}, Q_{2}(z) \geqq 0$. Also if these conditions obtain,

$$
P_{4}(x) \in T_{r}^{*}
$$

Theorem 5 is hardly a satisfactory answer for the case $m=4$ as the criterion involves a 4 th degree equation. However, it leads to the more cumbersome but intrinsically simpler conditions of the following theorems.

Theorem 6. Let $P_{4}(x) \in T^{*}$ and $a_{3}^{2}<4 a_{2}$. If $a_{3}<a_{0} a_{1}^{-1}$ or $a_{2}-4^{-1} a_{3}^{2}$ $>4^{-1} a_{1}^{2} a_{0}^{-1}, P_{4}(x) \in T_{i}$. Further if $a_{0} a_{1}^{-1}<2^{-1} a_{3}$ and $a_{2}-4^{-1} a_{3}^{2} \leqq 2 a_{1} a_{3}^{-1}$ $-4 a_{0} a_{3}^{-2}, P_{4} \in T_{r}^{*}$ and there are two factorizations or one according as

$$
a_{2} \geqq a_{1} a_{3}^{-1}-a_{0} a_{3}^{-2}
$$

is or is not valid.

Theorem 7. Let $P_{4}(x) \in T^{*}$ and $a_{3}^{2} \geqq 4 a_{2}$. If $a_{3}<a_{0} a_{1}^{-1}, P_{4}(x) \in T_{i}$.

(i) If $2^{-1} a_{3}>a_{0} a_{1}^{-1}, a N S C$ for two, one, or no factorizations of $P_{4}(x)$ within $T^{+}$is that both, just one, or neither of (5.1) and $a_{0}^{2} a_{1}^{-2}+a_{2}$ $\geqq a_{3} \cdot a_{0} \cdot a_{1}^{-1}$ hold.

(ii) If $r_{2}>a_{0} a_{1}^{-1} \geqq 2^{-1} a_{3}, P_{4}(x) \in T_{r}^{*}$ (with just one factorization) or $T_{i}$ according as (5.1) holds or not.

(iii) If $r_{2}=a_{0} a_{1}^{-1} \geqq 2^{-1} a_{3}$, then $P_{4}(x) \in T_{r}^{*}$ and there are two factorizations or one according as both (5.1) and $a_{1}^{4}>a_{0}^{2}\left[2 a_{0}-a_{1} a_{3}\right]$ hold or not.

Proof of Theorems 6 AND 7. By Theorem 5, a NSC that $P_{4}(x) \in T_{r}^{*}$ is that there exist $z$ in $\left(0, a_{3}\right]$ such that $z^{-2} P_{4}(-z)=0$ and $Q_{2}(z) \geqq 0$. But $z^{-2} P_{4}(-z)=Q_{2}(z)-R(z)$ where $R(z)=a_{1} z^{-1}-a_{0} z^{-2}$. Hence, it is NS that $Q_{2}(z)=R(z) \geqq 0$ for some $z$ in $\left(0, a_{3}\right]$. A consideration of the behavior of the graphs of these two functions yields the particularized results. The condition in (iii) arises from an examination of the slopes at $z=a_{0} a_{1}^{-1}$. Q.E.D.

The relationships among the coefficients $a_{j}$ are not exhausted by the considerations of the last two theorems. The remaining cases can 
be dealt with but the conditions obtained would be no easier to verify than that of Theorem 5 .

\section{REFERENCES}

1. A. Hurwitz, Über die Bedingungen unter welchen eine Gleichung nur Wurzeln mit negativen reelen Teilen besitzt, Math. Ann. vol. 46 (1895) pp. 273-284.

2. P. Levy, L'arithmetique des lois de probabilites, J. Math. Pures Appl. vol. 17 (1938) pp. 17-39.

3. T. S. Motzkin and I. J. Schoenberg, On lineal entire functions of complex variables, Proc. Amer. Math. Soc. vol. 3 (1952) pp. 517-526 and On quadral entire functions of complex variables, Bull. Amer. Math. Soc. vol. 58 (1952) p. 184.

4. P. Rosenbloom, Quelques classes de problemes extremaux, Bull. Soc. Math. France vol. 80 (1952) pp. 1-58.

5. J. V. Uspensky, Theory of equations, New York, McGraw-Hill, 1948.

Purdue University 